\title{
Studies on Douyin app Communication in Social Platforms: Take Relevant Douyin Short Videos and Posts on Microblog as Examples
}

\author{
Jing Li \\ Wuhan Sports University, Wuhan, Hubei, 430079, China
}

Keywords: Social media, Douyin short video, Microblog, Big data

\begin{abstract}
Short video is a new manifestation form integrating text and image records and it has become a new way of information record, transmission and sharing through tens of seconds or several mins' videos. Due to low production threshold, high readability and fast transmission speed, short video has gradually become a way for information transmission for new media users. Douyin short video is a music creative short video app, a product subordinated to Headlines Today, which entered into short video industry by means of "music + short video +socializing" with a latest valuation of USD 22 billion. On January 31, 2018, Douyin app topped Apple Store (free) shooting and recording list (free). Starting from data, this paper has collected 59508 posts on Microblog with "Douyin short video" as keywords through gsdata monitoring platform, and analyzed keywords in post contents for visual presentation through content analysis method, and tried to discover main contents and transmission modes of users when spreading Douyin short video in social media.
\end{abstract}

\section{Introduction}

In September $20^{\text {th }}$, Zhang Yiming, CEO of Headlines today announced to spend at least RMB 1 billion to subsidize short video creators in the upcoming one year. Since 2016, the platform content filed has transferred from text to image, and then animation and video. 2016 was a year of short video app explosion intensively. According to data from "2017 short video original advertisement white paper", users of short video are mainly males. Make users have exceeded female users, accounting for $55 \%$. Post 90 generation is the main force. Users under 30 years' old account for more than half of the total users, and mid-high end and above consumers account for 51.6\%. Traditional video platforms such as Headline Today, OMQQ, Baijiahao have distributed short video platform. Tencent invested Kuaishou at cost of USD 0.35 billion. Headlines today purchased North American music short video social platform Musical. Ly at the price of USD 1 billion and merged with Douyin short video. Short video becomes the trend of next content startup.

Short video develops quickly on social platforms due to simple production, low threshold, broad participation and strong sociality. Doudou short video focused on music industry and selected 15 seconds music climax part as user creation background music. Users can shoot and produce images according to music rhythm and lyrics. This paper has used Weibo as the research subject, web crawler tool to crawl Weibo contents about Douyin short video within five days. Through analysis on high-frequency words and contents in relevant posts, this paper will discuss characteristics of Douyin short video in social medial communication.

\section{Battle for Definition}

Each short video firms are defining short video so as to grasp the commanding height of industry standard by defining short video length. Each platform has their own rules of time duration. Weibo launched 15 seconds short video or several photos to compose 15 seconds' video, and defined short video as 15 seconds to explain time, site, task and users' original contents. Headlines today thinks 4 mins should be the duration most suitable for short video playing. On April $19^{\text {th }} 2017$, partner of Kuaishou Zeng Guangming issued the article "Kuaishou redefines short video: 57 seconds" and defined short video, i.e. vertical screen lasting 57 seconds. Kuaishou concludes it as the industrial 
standard of short video industry after judging and summarizing each behavior of more than 60 million users every day through AI system.

Short video emphasizes short but integrates text, image, business and comprehensive display plots as a story narration form for presenting a theme. The author defines short video based on its characteristics that it is a sound-picture 3D transmission mode based on social connection through content positioning in headline and storytelling in videos with a length of tens of seconds to several mins. According to Golden Second Award data held by Headlines Today in 2017, the average headline duration of Golden Second Award is 20.8 Chinese characters. The headline length is positively related to the video playing amount. Short videos with the duration of 25-30 characters has the highest average playing amount. In the era of function allocation, Headlines Today suggests that short video should pay more attention to headlines, attract flow through topic contents, attract audience to watch video stories and meet users' self-communication requirements through story narration and plot manifestation so as to improve distribution amount. In 2017, with the development of internet celebrity economy, short video has ushered into its development golden time, and started changing from UGC content production to PGC professional operation mode.

\section{Affect Producer with Rhythm and Music, Bring Resonance with Users}

According to ASOVIP data monitoring display, on January 31st 2018, Douyin short video topped among all app in Apple Store, exceeding WeChat and Taobao. It topped among shooting and recording app (free), exceeding Kuaishou. (Figure. 1).

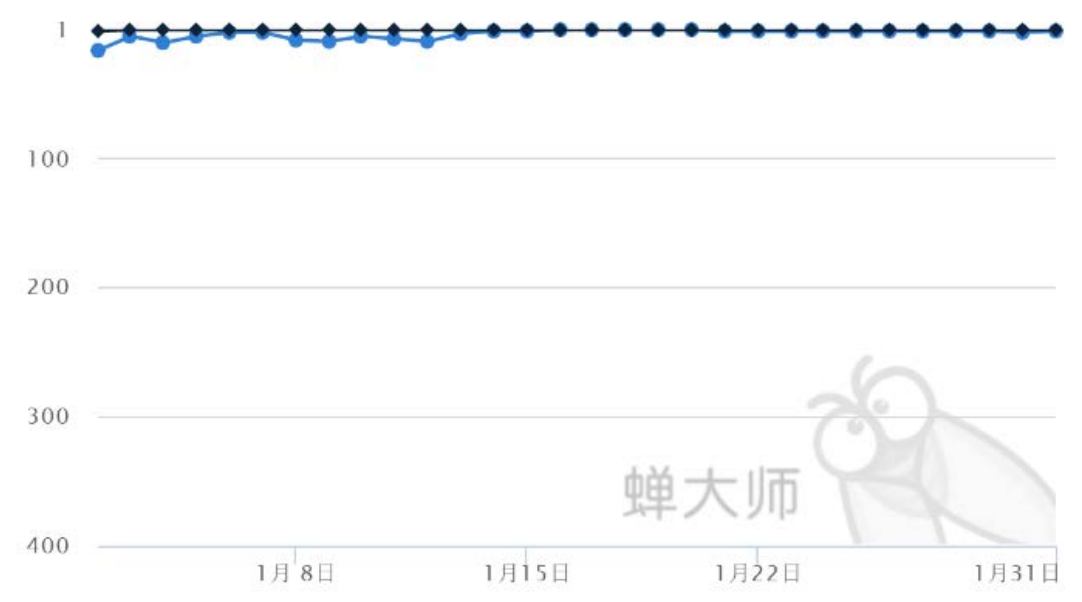

Figure. 1. Ranking of Douyin Short Video downloading in Apple Store

Douyin's characteristic is that producers do not only shoot images but choose music in APP and select music according to 25 music types such as pop, electrical music, American and European music. Then choose suitable rhythm and music to match suitable images. Hand dancing, body dancing, and funny lip-sync performance, and image recording should be included in image shooting. Image matching music with creative music performance as principal is the characteristic of Douyin. Its positioning is narrower comparing with Miaopai, Meipai, Kuaishou and other short video app but it mainly reflects producers' music creativity. Duration positioning within 15 seconds for producers to create attractive videos through dancing and editing has reflected the difficulty of APP and guaranteed music rhythm and controllable shooting contents within accurate time limit.

According to Baidu search index, users mainly search "music", "song", "most popular song in Douyin", "Douyin short video download", "Douyin short video website" when searing "Douyin short video" and show an increasing tendency (Figure 2). The author used web crawler tool with "Douyin short video" as keyword, collected 59508 relevant contents in Weibo within 5 days. According to word frequency analysis, "community" (4835 times), "music" (5341 times) are high-frequency words (Figure 3). The music lasting 15 seconds is the climax part in songs or currently popular songs, sections of most popular songs. Douyin platform reflects the most distinctive part of the rhythm of the 
whole song within 15 seconds. Positioning in music, Douyin make the music difficulty selectable so that users at all ages can use and make easy or difficult videos based on music. Pop music, electrical music, awesome music, classic songs, campus and brainwashing songs can meet young users' demands. Users can find inspiration from music by compiling dancing and editing pictures and produce creative music video works.

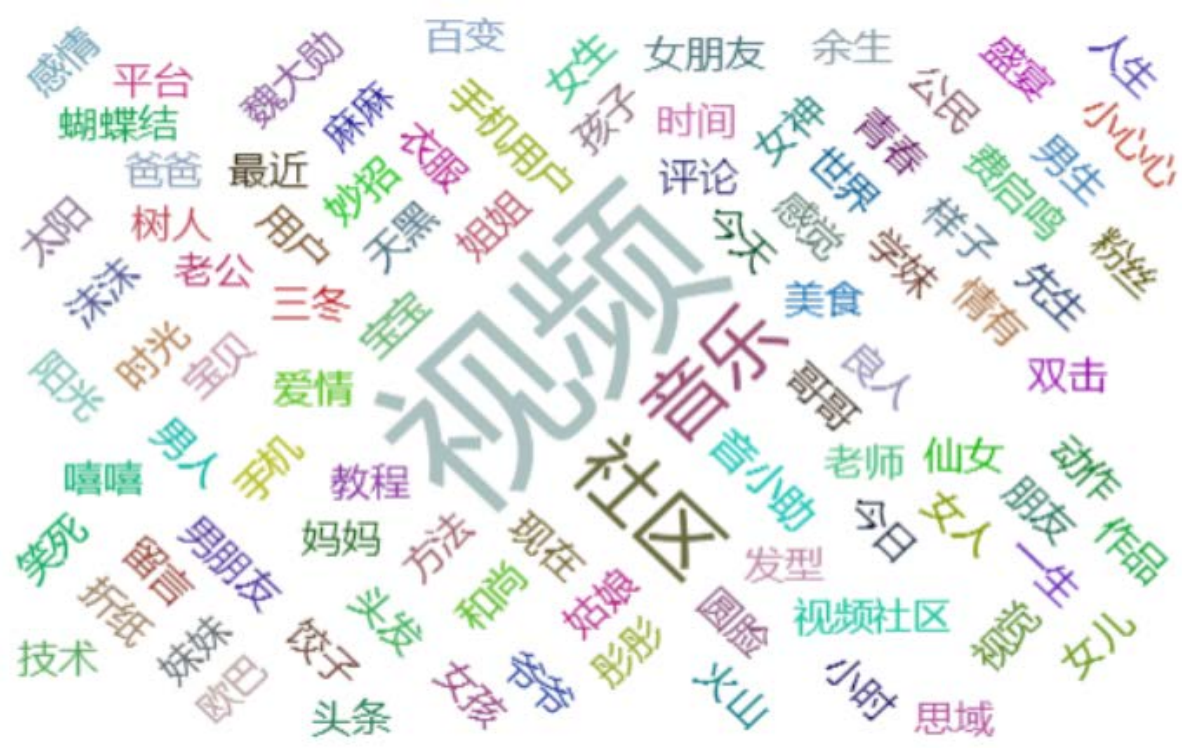

Figure. 2. Baidu search index of "Douyin Short Video"

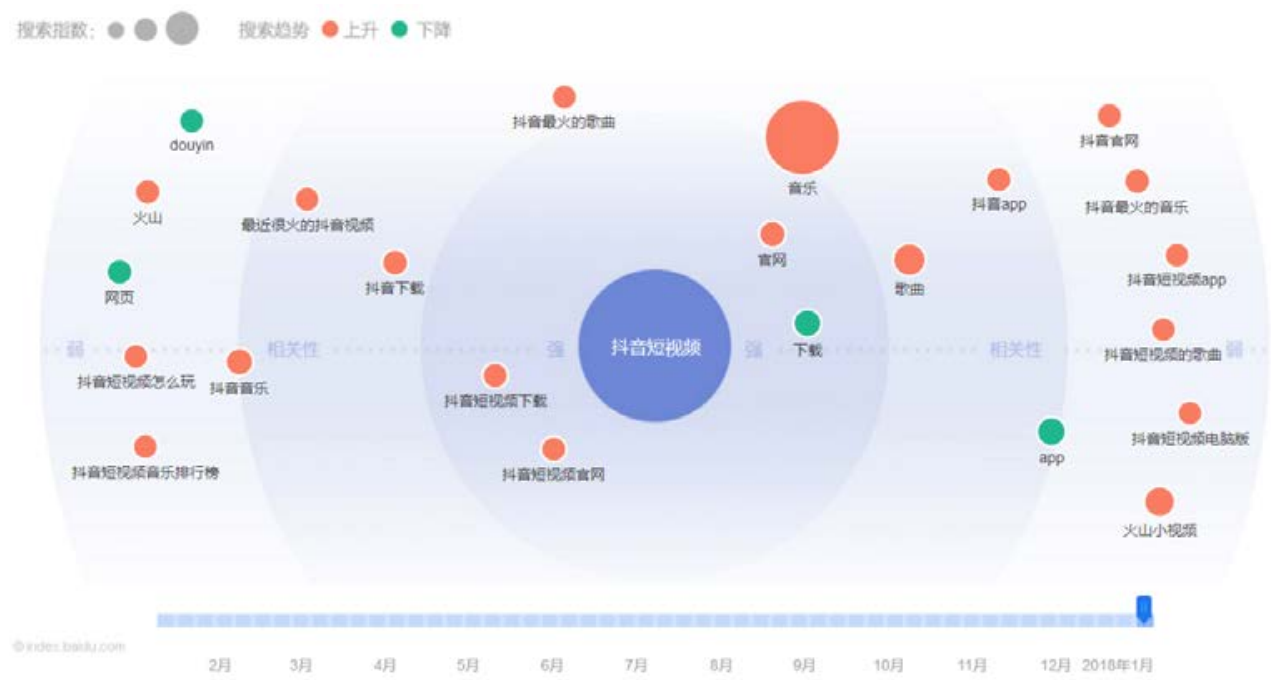

Figure. 3. Word cloud diagram of "Douyin Short Video"

\section{Reduce Users' Learning Cost and Motivate Transmission with Immersion}

Users can use segmented time when taking bus, waiting and break time to produce "short, flat and fast" videos for spiritual relaxation. They can also use leisure time to shoot relevant image with music for uploading to record life and share emotions. Seemingly professional music video production in Douyin has been well received by more users after lowering the technology threshold through video teaching. Users mention the keyword "Yinxiaozhu" (2081 times) while sharing videos on Webo. Douyin shooting assistant or Douyin shooting guideline is the section for users to learn music video recording and to set the topic as \#Douyin shooting guideline". When new users see the cool video and would like to shoot without knowing how, they can shoot a wonderful short video, obtain the uploading qualification and issue the video. Then they can search or tweet the courses about this topic for self-learning. According to Weibo contents, there are numerous topics about this shared in social platforms. Users mainly show their skills and communicate with each other on social platforms 
through the keyword "Yinxiaozhu".

Douyin has won the victory with content in social level, through which users can express contents firstly and tweet more with more points in excellent videos. Different with live broadcasting, E-business live, Douyin video mainly attract users' click with video quality instead of by following. Users will be given points if they like and then more users will see and like it. User click points to show that they love it and then simple heart beating effect will appear in the picture. Then double click the screen to show they like it more, which can bring strong visual impact to users so that they are immersed in the atmosphere of watching and evaluation. According to the newest comment area display, the fall-glide loading in the platform can help users to focus on the video rather than text comments. Picture theme, issuer's name, video description, topic, and current background music are clear in the screen. Users decide social sharing or not according to information on the screen when watching the video, and they can forward for topic secondary discussion after sharing. Decentration in mobile era is more and more obvious which makes users share contents at any time anywhere through social media. According to Weibo contents, users will forward together with their comments while forwarding topics with "\#" so that their music videos can attract more attention and clicks.

\section{Short Video Attempts Realization and Transformation and Enters into "+" Era}

In the era of internet celebrity, short video is the essential communication weapon for internet celebrities. At the advent of shot video + e-business era, with the sharp increase of Douyin short video users, micro merchants and e-business use the social platform to implant product directly such as PPT production teaching, food making methods, English teaching, music teaching and dance teaching, etc. Through music video presentation, users can watch vividly. The direct expression form of short video, diverse contents and strong interaction have brought more profitable modes. Through individualized video and visual and sound impact from pop music such as segmented form and content breakdown, users' attention and purchase desire are stimulated. Short video +tourism, short video+ teaching, short video + food are popular subdivision on short video platform. Douyin short video has shown a tendency of music courses, dance course and other micro merchant subdivision. Meanwhile, internet celebrities have a large amount of fans who can improve views and subscription after settling in Douyin and attract advertisement merchants and investors with current fans.

Except for realization, short video attempts change in its form and contents by providing other video creativities for users with other contents such as "+" sports contest except for music creative performance on platforms. Federation of University Sports of China has issued "the $19^{\text {th }}$ CUBA Cheerleading Qualification Contest Rules" and announced that contestants used Douyin app to record creative and high-quality video contents in base competition and division competition, then @ CUBA cheerleading babies for vote supporting. In division competition, contests are required to solicit votes online and offline marking \#entering into final eight" with the creation contents combining with the competition court, and share to social platforms through Douyin App. The competition holder indicated that Douyin and CUBA official should provide teaching instruction. "+" sports is a new attempt of Douyin transformation. Users can make and share with a competition theme as creative point so as to bring benefits to Douyin and the competition.

\section{Conclusions}

Young people are the major user markets for short video. Douyin short video has located the professional entry point to attract young people who loves new things through music creative video. The form has broken the board between consumers and products, and meanwhile innovated interactive scene mode, and create new social experience for young generation popular culture. The text contents crawled show that users hope for more discussion shooting skills. So Douyin should start up live broadcasting function in social connection and enrich its interface so as to bring better user experience. 


\section{References}

[1] China Short Video Market Research Report of First Half Year of 2017, see http://www.iimedia.cn/56105.html.

[2] Liu Guoqiang. "Web space collective action as interactive ritual, Journal of International Communication, the 11th issue.

[3] Wang Xiaohong, Discussion on web video discourse, Modern Communication, 2nd issue, 2013.

[4] Huang Zhengmao, "Method of popularity of the No.1 internet celebrity Papi in 2016---From the perspective of audience use and satisfaction, West China Broadcasting TV, 9th issue, 20016.

[5] Gao Cong, Yang Boxu, Analysis on micro video content production mode-studies on Miaopai, Sina Weibo official short video app, Press Circles, 23rd 2016. 